\title{
Cidades inteligentes: insights e contribuições das pesquisas brasileiras
}

\author{
Smart Cities: Insights and Contributions from Brazilian Research
}

Kellen Lazzaretti[a] (iD) Simone Sehnem [a] (iD) Fernando Fantoni Bencke[a] (iD) Hilka Pelizza Vier Machado[a] (iD)

[a] Universidade do Oeste de Santa Catarina (UNOESC), Chapecó, SC, Brasil

Como citar: Lazzaretti, K., Sehnem, S. \& Bencke, F. F., Machado, H. P. V. (2019). Cidades inteligentes: insights e contribuições das pesquisas brasileiras. urbe. Revista Brasileira de Gestão Urbana, v. 11, e20190118. DOI https://doi.org/10.1590/21753369.011.e20190118

\section{Resumo}

O objetivo deste estudo foi identificar a rede de pesquisadores brasileiros que estudam cidades inteligentes e descrever as principais contribuições para o debate sobre o tema. Foi realizada a representação de redes de relacionamento para obter uma fotografia dos núcleos de pesquisa em "cidades inteligentes" e uma revisão sistemática da literatura das produções nacionais existentes. Os resultados demonstram que há uma predominância conceitual e empírica de cidades inteligentes que reúnem aspectos relacionados à Tecnologia da Informação e Comunicação e à qualidade de vida das pessoas, conectando-as, capacitando-as, a fim de promover interações constantes nas cidades e governança pública. Pesquisadores que estudam o assunto estão localizados principalmente na USP, PUC Campinas, UFPE e UNINOVE, no entanto, não foram evidenciadas redes de relações entre universidades na condução da pesquisa. 0 artigo auxilia na análise geral da pesquisa realizada no Brasil sobre cidades inteligentes, bem como, contribui para a discussão da necessidade de uma visão mais ampla do conceito de cidades inteligentes, que vai além da criação de TICs evidenciado neste estudo.

Palavras-chave: Cidades inteligentes. Redes. UCINET.

\section{Abstract}

The aim of this study was to identify the network of Brazilian researchers studying smart cities and describe their main contributions to the debate on the theme. Representation of relationship networks was carried out to obtain a photograph of the nuclei of research in "smart cities" and a systematic review of the literature of the existing national productions. The results show that there is a conceptual and empirical predominance of smart cities that bring together aspects related to Information and Communication Technology and people's quality of life, connecting them and enabling them in order to promote constant interactions in cities and public governance. Researchers who study the subject are located mainly at USP, PUC Campinas, UFPE and

KL é mestre em Adminstração, e-mail: kellen.lazzaretti29@gmail.com

SS é doutora em Administração, e-mail: simone.sehnem@unoesc.edu.br

FFB é doutor em Administração, e-mail: fernando.bencke@unoesc.edu.br

HPVM é Doutora em Engenharia de Produção, e-mail: hilka.machado@unoesc.edu.br 
UNINOVE, however, networks of relations between universities in the conduction of the research were not evidenced. The article assists in the general analysis of the research carried out in Brazil on intelligent cities as well as contributes to the discussion of the need for a broader view of the concept of intelligent cities, which goes beyond the creation of ICTs evidenced in this study.

Keywords: Smart cities. Networks. UCINET.

\section{Introdução}

Cidades são consideradas sistemas complexos caracterizados por um grande número de cidadãos interconectados, empresas, diferentes meios de transporte, redes de comunicação, serviços e utilidades. 0 crescimento populacional e o aumento da urbanização elevam uma variedade de problemas técnicos, sociais, econômicos e organizacionais que tendem a comprometer a sustentabilidade econômica e ambiental das cidades (Neirotti et al., 2014).

Nesse contexto surgiram debates sobre o modo como as novas soluções baseadas em tecnologia, bem como novas abordagens para o planejamento e a vida urbana, podem assegurar a viabilidade e a prosperidade futuras em áreas metropolitanas (Neirotti et al., 2014). Expressões como "cidades criativas", "cidades sustentáveis" e "cidades inteligentes" têm ganhado cada vez mais espaço nas agendas públicas e na literatura.

Um dos marcos da ideia de smart cities surgiu no Fórum Mundial de 1997 sobre Cidades Inteligentes em que cerca de 50 mil cidades e vilas ao redor do mundo desenvolveriam iniciativas inteligentes para a próxima década (Holland, 2008). E nesses 20 anos o conceito de "cidade inteligente" foi introduzido como um dispositivo estratégico para abranger os fatores de produção urbana moderna em uma estrutura comum e para destacar a importância das TICs para melhorar o perfil competitivo de uma cidade (Caragliu et al., 2011). Outra aliada na construção do conceito de cidades inteligentes é a Internet das Coisas urbanas, uma infraestrutura de comunicação que oferece acesso unificado, simples e econômico a uma infinidade de serviços públicos, desencadeando potenciais sinergias e aumentando a transparência para os cidadãos (Zanella et al., 2014).

Tornar uma cidade inteligente está emergindo como uma estratégia para mitigar os problemas gerados pelo crescimento da população urbana e pela rápida urbanização, no entanto, pouca pesquisa acadêmica tem discutido com profundidade o fenômeno (Chourabi et al., 2012).

Embora os estudos que exploram cidades inteligentes estejam presentes desde 1999 nas bases de dados consultadas, foi apenas a partir de 2012 que ganharam maior representatividade em número de publicações listadas na Scopus, por exemplo (Pinheiro Junior \& Cavalheiro, 2017). No Brasil, há poucos estudos acadêmicos ou aplicados, os existentes são de áreas interdisciplinares, como administração, sistemas de informação, engenharias, arquitetura, políticas públicas e outras, e possuem um alinhamento com as pesquisas internacionais em termos de modelos, utilizando frameworks dos autores Chourabi et al. (2012) e Nam e Pardo (2011) (Pinheiro Junior \& Cavalheiro, 2017). Entende-se que ao construir uma rede social de pesquisadores é possível ter uma fotografia do campo e assim ter mais clareza de que forma os pesquisadores brasileiros tem contribuído para o desenvolvimento do tema.

A análise de redes sociais pode ser definida como a padronização das relações existentes entre determinados atores sociais em diferentes níveis de análise e, estudar as relações sociais entre atores é fundamental para a ciência social (Breiger, 2004). Diante do exposto, este estudo objetiva identificar a rede de pesquisadores brasileiros que estudam cidades inteligentes e analisar suas principais contribuições para o debate sobre o tema.

Um estudo anterior foi realizado por Pinheiro Junior e Cavalheiro (2017), cujo objetivo foi propor uma agenda de estudos sobre as cidades inteligentes no Brasil, a partir de uma revisão da literatura sobre o tema. Os autores analisaram publicações nacionais e realizaram um alinhamento das pesquisas brasileiras sobre a área com o contexto internacional. A principal contribuição do nosso estudo consiste no mapeamento dos pesquisadores brasileiros e a sinalização dos potenciais de formação de redes para a consolidação do tema no Brasil. 
Este artigo está dividido em cinco partes, na introdução apresentamos o objetivo e justificativa do estudo, na segunda elaboramos uma revisão bibliográfica sobre tema cidades inteligentes, na terceira apresentamos os procedimentos metodológicos, na quarta parte apresentamos os resultados, as redes sociais identificadas e as contribuições dos estudos realizados. Na quinta parte discutimos os dados, conclusões e sugerimos estudos futuros na área.

\section{Cidades inteligentes}

Embora ainda não exista uma definição consensual e amplamente aceita de cidades inteligentes, seu objetivo final é promover melhor uso dos recursos públicos, aumentando a qualidade dos serviços oferecidos aos cidadãos, enquanto reduz os custos operacionais da administração pública (Zanella et al., 2014). Segundo Neirotti et al. (2014) tendências atuais e os padrões de evolução de cidade inteligente dependem, em grande parte, dos fatores contextuais locais, que dizem respeito aos recursos naturais e energia, transporte e mobilidade, edifícios, vida, governo, economia e pessoas.

Uma cidade é considerada inteligente quando os investimentos em capital humano e social, infraestrutura de comunicação tradicional (transporte) e moderna impulsionam o crescimento econômico sustentável e uma alta qualidade de vida, com uma gestão inteligente dos recursos naturais, por meio de governança participativa (Caragliu et al., 2011). A maioria das iniciativas de cidades inteligentes é impulsionada pelos governos, mas alavancada pelo uso das TICs para melhor servir aos cidadãos (Chourabi et al., 2012). Uma cidade na qual as TICs se fundem com as infraestruturas tradicionais, coordenadas e integradas usando novas tecnologias digitais são consideradas inteligentes (Batty et al., 2012).

Uma cidade inteligente possui algumas características que a diferenciam das demais, como: (i) a utilização da infraestrutura de rede para melhorar a eficiência econômica e política e permitir o desenvolvimento social, cultural e urbano (Hollands, 2008); (ii) possui uma ênfase subjacente no desenvolvimento urbano conduzido pelos negócios (Hollands, 2008); (iii) um forte foco no objetivo de conseguir a inclusão social de vários residentes urbanos em serviços públicos (Caragliu et al., 2011); (iv) ênfase no papel crucial das indústrias de alta tecnologia e criativas no crescimento urbano de longo prazo (Caragliu et al., 2011); (v) uma profunda atenção ao papel do capital social e relacional no desenvolvimento urbano (Caragliu et al., 2011); e (vi) a sustentabilidade social e ambiental como um componente estratégico importante (Caragliu et al., 2011).

Chourabi et al. (2012) sugerem uma estrutura que pode ser usada para caracterizar uma cidade inteligente e projetar iniciativas que promovam essa visão, composta por fatores externos como governança, pessoas e comunidades, ambiente natural, infraestrutura e economia e fatores internos como tecnologia, gestão e política. Contudo a tecnologia pode ser considerada como fator que, de alguma forma, influencia todos os outros fatores de sucesso na estrutura, devido ao fato de que muitas iniciativas de cidades inteligentes estejam utilizando intensivamente a tecnologia.

Uma aliada no desenvolvimento de cidades inteligentes é a Internet das Coisas urbanas (IoT). As IoTs urbanas são projetadas para apoiar a visão smart city, que visa explorar as mais avançadas tecnologias de comunicação para apoiar serviços de valor agregado para a administração da cidade e para os cidadãos (Zanella et al., 2014). A grande implantação da IoT está, na verdade, possibilitando projetos e iniciativas de cidades inteligentes em todo o mundo (Hammi et al., 2018). A IoT traz um novo conceito para cidades inteligentes como o descrito por Byun et al. (2016). São cidades que estão embasadas na construção de uma rede de comunicação entre M2M (Machine to Machine), IoT e IoE (Internet of Everything), amparadas em uma economia criativa, aonde é enfatizada a sua realização pelos dos governos.

Para um desenvolvimento eficaz de cidades inteligentes, Berst (2018) ressalta que há necessidade de uma visão sistêmica mais apurada e que a falta dela pode fazer com que aqueles que pensam as cidades inteligentes escolham as prioridades erradas, criem soluções fragmentadas que estão presas no que ele chama de silos departamentais e não consigam capturar sinergias (oportunidades de compartilhar infraestrutura, custos e dados). Berst (2018) ainda critica que muitos governos ainda pensam nos cidadãos como habitantes e não como clientes. Em uma cidade inteligente o conceito de cidadão se torna muito limitado, pois as cidades recebem turistas, trabalhadores, negociadores, investidores, dirigentes de outras 
cidades e, assim, há uma competição por esses clientes com todas as outras cidades que querem atrair empregos, talentos e turistas (Berst, 2018).

\section{Procedimento metodológico}

O estudo foi realizado utilizando a representação de redes. Uma rede é entendida como um grupo de indivíduos que, de forma agrupada ou individual, relacionam-se com outros com um fim específico caracterizado por um fluxo de informações (Alejandro \& Norman, 2005).

Estudos de análise de redes sociais ampliaram o foco de grupos pequenos para grupos em larga escala. Essa mudança deu-se em função da maior disponibilidade de dados sociais por meio de ferramentas digitais e também pela criação de métodos computacionais que auxiliam aos pesquisadores na coleta e análise dos dados (Berst, 2017). Para auxiliar aos pesquisadores na construção e análise de redes de relacionamento foi utilizado o software UCINET 6.109®.

A coleta de dados foi realizada nos meses de novembro e dezembro de 2018. Na busca realizada na Biblioteca Digital Brasileira de Teses e Dissertações (BDTD) utilizamos o termo "Cidades Inteligentes" e localizamos 104 publicações nas mais diversas áreas do conhecimento. Contudo, quando realizamos a leitura dos documentos, observamos que um deles era um trabalho de conclusão de graduação com dois autores listados, uma dissertação não estava disponível e outra não foi localizada a autoria. Com isso, restaram 100 documentos para análise. Com a busca foi possível ter acesso a dados do autor, orientador, instituição de ensino e programa ao qual o pesquisador estava vinculado para a construção das redes.

Foi realizada busca na Biblioteca Eletrônica de Periódicos Científicos (Spell) com as palavras-chave "cidades inteligentes" localizadas no resumo, desse modo, foram localizados 12 artigos científicos. É importante destacar que o Spell é uma base focada em publicações na área de gestão, logo, publicações em outras áreas não foram contempladas.

Outra fonte de coleta de dados foi a Plataforma Lattes do CNPq. Nesta realizamos busca por grupos de pesquisa com o termo "Cidades Inteligentes" no dia 05 de novembro de 2018 e foram localizados 83 grupos, seus pesquisadores e universidades as quais estavam ligados, permitindo também a construção de redes de relacionamento.

Em um segundo momento houve a leitura dos resumos, introdução e conclusão das 100 teses e dissertações localizadas e dos 12 artigos a fim de realizar uma análise sistemática. Foram coletados dados como tema, objetivo, contribuições e sugestões para estudos futuros.

Foram propostas as seguintes categorias de análise: universidade, autor, programa e grupo de pesquisa originário do autor, tema, problema, contribuições das produções científicas. Com essas categorias, mapas de redes e análise sistemática das produções foi possível propor um panorama de pesquisa em cidades inteligentes no Brasil.

\section{Resultados}

Redes de relacionamento entre instituições, grupos de pesquisa e pesquisadores 
Consideramos importante em nosso estudo conhecer os grupos de pesquisa em cidades inteligentes e as universidades aos quais estão vinculados. Um grupo de pesquisa é um indicativo de fortalecimento do tema naquela instituição de ensino e de uma rede de pesquisadores que ajudam a desenvolver a pesquisa brasileira em cidades inteligentes.

Foi possível identificar 83 grupos de pesquisa distribuídos em 58 Universidades diferentes em todo o Brasil. A rede completa de grupos e universidades pode ser observada na Figura 1.

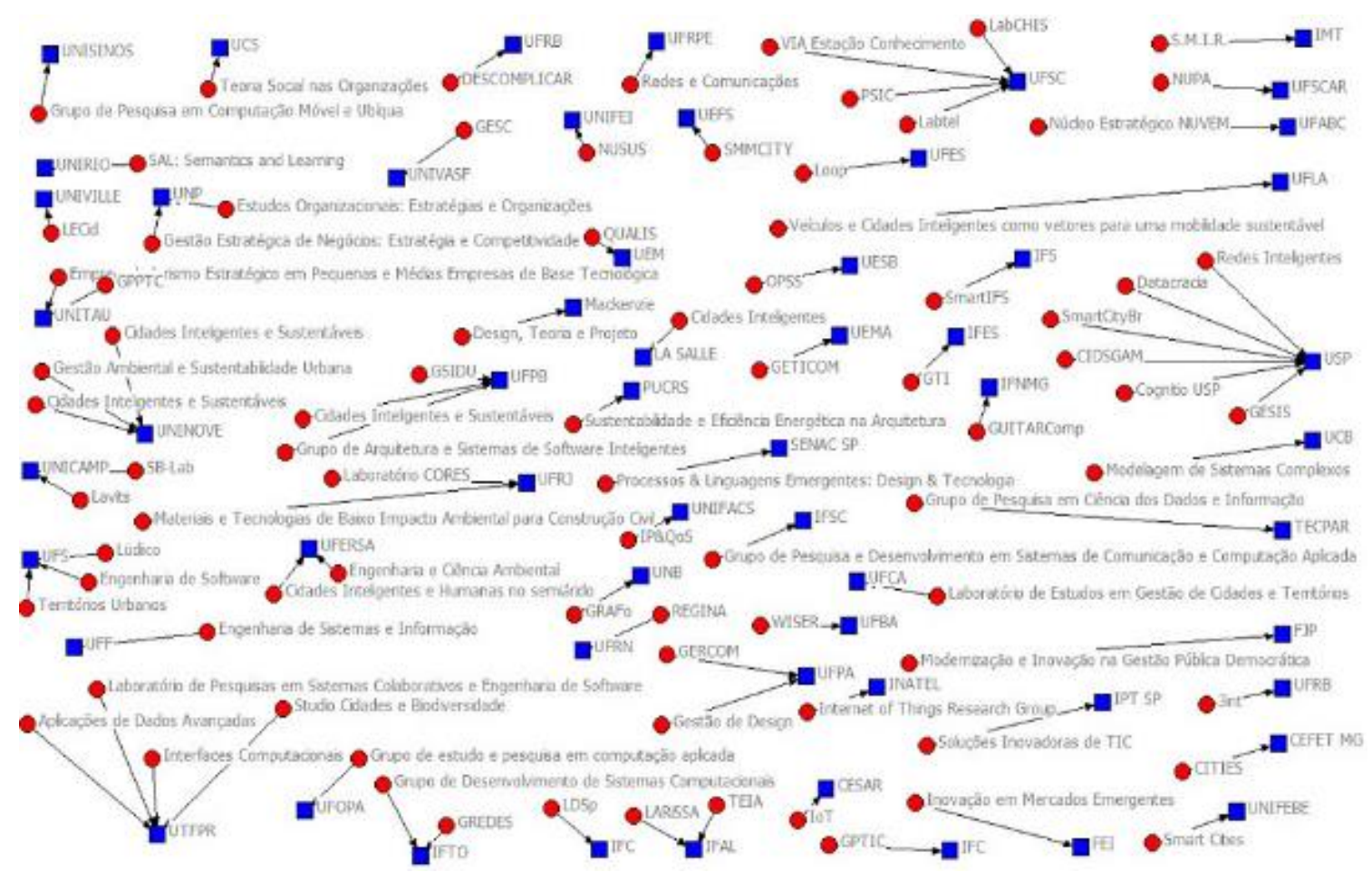

Figura 1 - Redes sociais universidades e grupos de estudo em cidades inteligentes Fonte: Elaborado pelos autores com auxílio do software UCINET (2018).

A instituição de ensino com o maior número de grupos de pesquisa em cidades inteligentes é a Universidade de São Paulo (USP), que conta atualmente com sete grupos de pesquisa na área. Esses grupos possuem linhas de pesquisa distintas nas áreas de Ciências Sociais Aplicadas e Ciências Exatas e da Terra. Na linha de pesquisa em Ciências Sociais Aplicadas têm-se os seguintes grupos: (1) Cidade, Sustentabilidade e Gestão Ambiental (CIDSGAM), formado em 2011 e conduzido pelos professores Prof. Dr. Paulo Santos de Almeida e Prof. Dr. Marcio Henrique da Costa Gurgel; (2) Datacracia, formado em 2016 e liderado pelo Prof. Dr. Luiz Guilherme de Carvalho Antunes; (3) Grupo de Estudos em Sistemas, Inovação e Sustentabilidade (GESIS), formado em 2017 o grupo é liderado pela Prof. ${ }^{\text {a }}$ Dr. ${ }^{\text {a }}$ Lara Bartocci Liboni; (4) Pesquisa em Cidades

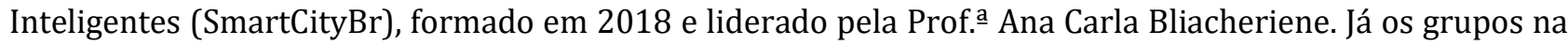
linha de pesquisa de Ciências Exatas e da Terra são (5) Computação Cognitiva (Cognitio USP), este grupo existe desde 2003; (6) Computação Paralela e Distribuída coordenado pelo Prof. Dr. Siang Wun Song; e (7) Redes Inteligentes, formado em 2017 e liderado pela Prof. ${ }^{a}$ Kalinka Regina Lucas Jaquie Castelo Branco.

A Universidade Federal de Santa Catarina (UFSC) possui quatro grupos de pesquisa que desenvolvem estudos em cidades inteligentes: (1) na área de Engenharia o grupo Habitats de Inovação e Empreendedorismo (VIA Estação Conhecimento) liderado pela Prof.모 Dr.므 Clarissa Stefani Teixeira e (2) 
Labtel liderado pelo Prof. Dr. Roderval Marcelino. Na área de Ciências Sociais Aplicadas destaca-se o (3) Laboratório de Cidades mais Humanas, Inteligentes e Sustentáveis (LabCHIS), liderado pelo Prof. Dr. Eduardo Moreira da Costa, e na área de Ciência Humanas destaca-se o grupo (4) Psicologia, Subjetividade, Inovação e Conhecimento (PSIC), coordenado pelo professor Dr. Silvio Serafim da Luz Filho.

A Universidade Tecnológica Federal do Paraná (UTFPR) e a Universidade Federal de Sergipe (UFS) possuem também quatro grupos de pesquisa cada um nas áreas de Ciências Sociais Aplicadas, Ciências Exatas e da Terra. As demais universidades possuem pelo menos um grupo relacionado ao tema. A Universidade Tecnológica Federal do Paraná (UTFPR) tem os grupos de pesquisa: (1) Aplicações de Dados Avançadas/Advanced Data Applications; (2) Interfaces Computacionais; (3) Laboratório de Pesquisas em Sistemas Colaborativos e Engenharia de Software; e (4) Studio Cidades e Biodiversidade. Já a Universidade Federal de Sergipe (UFS), destaca-se os grupos: (1) Engenharia de Software; (2) Laboratório para Universalização do Desenvolvimento, Inovação e Inteligência (Lüdiico); (3) Tecnologias em Arquitetura e Urbanismo; e (4) Territórios Urbanos.

A Figura 2 ilustra as redes de relacionamento entre autores e universidades identificadas a partir das publicações disponíveis na Biblioteca Digital Brasileira de Teses e Dissertações (BDTD). Os nós mais densos visualizados são congregados pela USP, FGV e UNINOVE, que possuem o potencial de congregar o maior número de autorias.

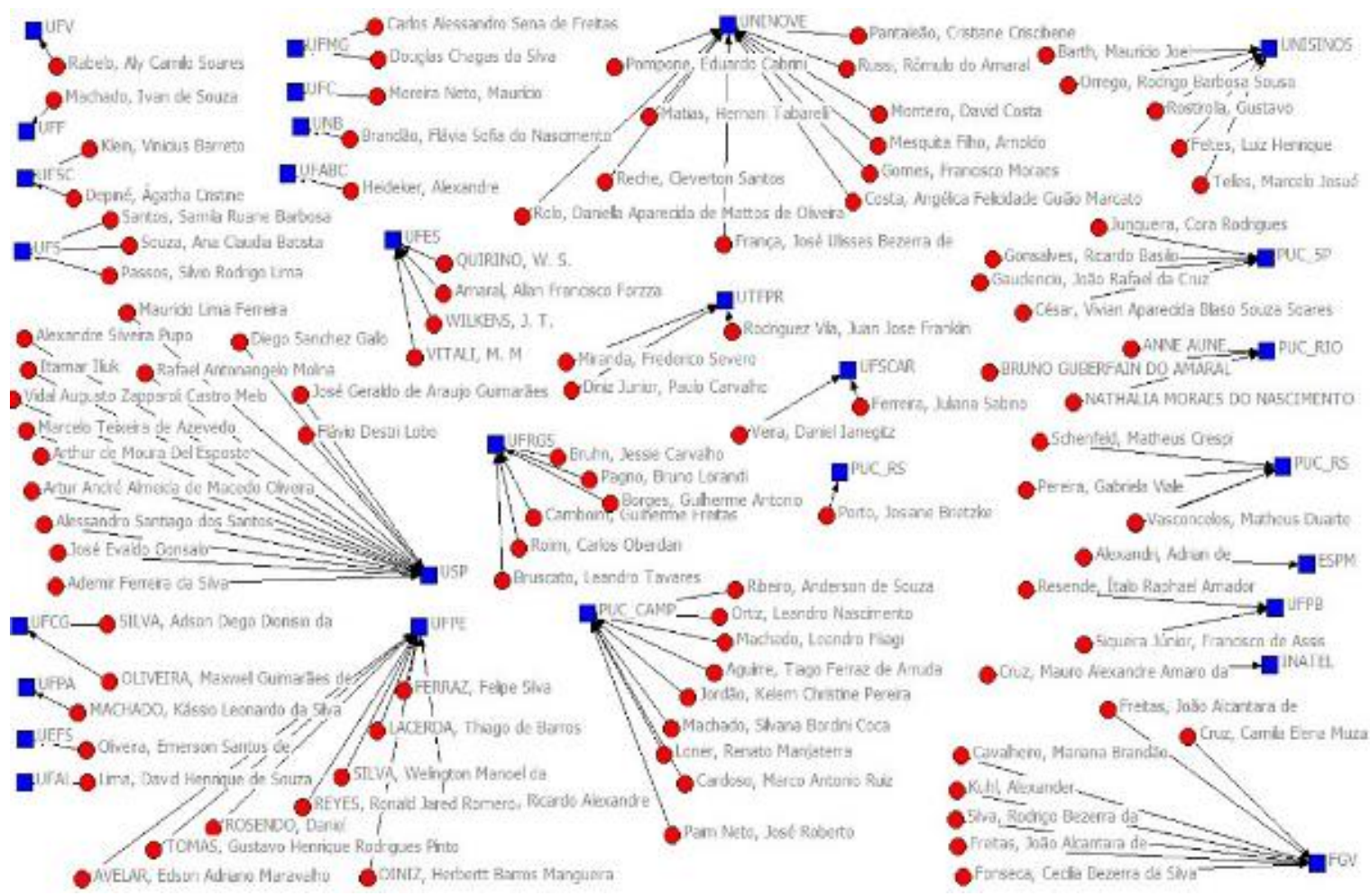

Figura 2 - Redes sociais universitárias e autores de teses e dissertações sobre cidades inteligentes Fonte: Elaborado pelos autores com auxílio do software UCINET (2018).

Ao analisarmos as publicações em periódicos nacionais constantes na Spell localizamos 12 publicações cujo tema é cidades inteligentes, contudo, não foi possível construir uma rede com esses estudos, pois não há coautorias entre os autores. Também, observamos que, entre os periódicos 
identificados estão àqueles pertencentes às universidades com maior número de produções e grupos de estudo na área, com destaque para Universidade Federal de Pernambuco (UFPE) - Revista Eletrônica de Gestão Organizacional (GESTÃO.Org) - e Universidade Nove de Julho (UNINOVE) - Internacional Journal of Innovation. Contudo, o maior número de publicações pertence à Revista Eletrônica de Sistemas de Informação pertencente ao Instituto Brasileiro de Estudos e Pesquisas Sociais (IBEPES).

A partir dos dados analisados, podemos identificar universidades que se destacam na produção de pesquisa acerca de cidades inteligentes, como a USP, UNINOVE, FGV, PUC Campinas e UFPE. Essas universidades possuem programas de mestrado diversos que pesquisam cidades inteligentes sob diversas perspectivas. A USP desenvolve seus estudos mais ligados às áreas de Administração e Engenharia Elétrica; a FGV na área de gestão; a PUC Campinas tem desenvolvido esses estudos no programa de mestrado em Engenharia Elétrica; na UFPE estão ligados aos estudos na área de Ciências da Computação; e a UNINOVE possui um Programa de Mestrado em Cidades Inteligentes e Sustentáveis (PPG-CIS). Este programa tem como propósito a compreensão da dinâmica socioespacial do território urbanizado e seus problemas intrínsecos a fim de oferecer pesquisas que tragam propostas para o enfrentamento dos desafios (UNINOVE, 2019). O PPG-CIS possui três linhas de pesquisa: (1) regulação indutora e instrumentos urbanos; (2) espaços urbanos, sociedade civil de democrática; e (3) inovação aplicada ao planejamento urbano. A partir dos dados coletados podemos considerar que as redes de relacionamento existem nos grupos de pesquisa em cada universidade, contudo, não observamos redes de relacionamento entre universidades para a produção de pesquisa em cidades inteligentes.

\section{Análise das produções brasileiras sobre cidades inteligentes}

As áreas de Ciências da Computação (23 publicações) e Engenharia Elétrica (20 publicações) se sobressaem nas pesquisas sobre o tema. A UNINOVE também possui um PPG específico em Cidades Inteligentes e Sustentáveis e já gerou 11 dissertações na área. Administração também aparece como destaque nas áreas em que são desenvolvias publicações sobre o tema com nove dissertações e teses.

Evidenciamos a diversidade de autores utilizados para conceituar cidades inteligentes, pois poucos autores brasileiros arriscaram em cunhar um conceito próprio, adotando assim conceitos de autores internacionais. Contudo, muito embora não haja consenso entre as definições conceituais de cidades inteligentes, há um predomínio conceitual de definições que congregam aspectos associados à Tecnologia da Informação e Comunicação (TIC) e qualidade de vida das pessoas, conectando-as, empoderando-as, de modo a promoverem constantes interações nas cidades e auxiliando na governança pública. Ao que indicam os estudos, seu foco principal parece ser o papel da infraestrutura de Tecnologias de Informação e Comunicação (TICs), embora muitas pesquisas também tenham sido realizadas sobre o papel do capital humano, educação, capital social e relacional e interesse ambiental como importantes para o crescimento urbano (Caragliu et al., 2011). Entendemos que as soluções baseadas em TIC podem ser consideradas apenas como um dos vários recursos de entrada para projetos e abordagens para o planejamento urbano e de vida. Iniciativas baseadas em TICs não tornam uma cidade necessariamente "inteligente", serão apenas 
produções intermediárias que refletem os esforços feitos para melhorar a qualidade de vida dos cidadãos (Neirotti et al., 2014).

As soluções em TICs, contudo, são importantes aliadas à governança pública. No Brasil o Decreto $\mathrm{n}$. 9.203, de 22 de novembro de 2017 define governança pública como "conjunto de mecanismos de liderança, estratégia e controle postos em prática para avaliar, direcionar e monitorar a gestão, com vistas à condução de políticas públicas e à prestação de serviços de interesse da sociedade" (Brasil, 2017, p. 1). Nesse ínterim, para que a governança pública seja mais efetiva, a utilização de TICs faz-se necessária, uma vez que ela tem como princípios: (i) capacidade de resposta; (ii) integridade; (iii) confiabilidade; (iv) melhoria regulatória; (v) prestação de contas e responsabilidade; e (vi) transparência.

As 11 diretrizes dispostas no Decreto n. 9.203, de 22 de novembro de 2017 também fortalecem e evidenciam a necessidade de desenvolvimento de tecnologias para facilitar a gestão pública e conduzir ao alcance dessas diretrizes, principalmente no que tange a integração de dados entre as esferas públicas para melhor tomada de decisão, melhoria de controles internos e fornecimento de acesso público à informação.

Para a realização dessas pesquisas alguns municípios brasileiros serviram de objetivo de estudo: Búzios (RJ), Belo Horizonte (MG), Rio de Janeiro (RJ), Gonçalves (MG), Curitiba (PR), Campinas (SP), São Paulo (SP), Poá (SP), Cabreúva, Santana do Parnaíba e São José do Rio Preto (SP), Taguatinga (DF), Barbalha, Mauriti e Maranguape (CE) e Roma (IT). Evidenciamos, contudo, que alguns desses municípios como Búzios, no Estado do Rio de Janeiro, e São Paulo capital foram objeto de mais de um estudo. No caso de Búzios o que motivou os estudos foram os projetos existentes no município com vistas para torná-lo uma cidade inteligente, como o projeto de energia elétrica iniciado em 2011 e desenvolvido pela concessionária Ampla S.A., que tem como proposição transformar Búzios na primeira cidade inteligente da América Latina. 0 projeto da concessionária é basicamente pautado em melhorias da rede elétrica, o que seria apenas um dos elementos de um projeto de cidade inteligente (Freitas, 2014).

0 Rio de Janeiro é outra cidade que serviu como objeto de estudo. Em 2010 foi inaugurado o Centro de Operações Rio (COR), uma sala de controle que monitora todas as câmeras da prefeitura e reúne sob o mesmo teto os representantes de cerca de 30 agências municipais, concessionárias que atuam na cidade e forças de segurança com o objetivo de criar um protocolo de ação e diminuir o tempo de respostas para eventuais emergências. (Freitas, 2018). Belo Horizonte e Porto Alegre também possuem Centro de Operação, o que motivou estudos acadêmicos (Pereira, 2016). No caso de São Paulo, além de ser a maior cidade do Brasil, é considerada uma das cidades inteligentes da América Latina. Estudo foi desenvolvido com o intuito de estudar um sistema para redução de custo de energia em linhas de metrô, pois o setor de transporte de São Paulo é um dos maiores consumidores de energia do município (Gomes, 2017). De uma forma geral as cidades utilizadas como objeto nos estudos analisados foram escolhidas, basicamente, por quatro motivos macro: (1) porque possuem algum projeto para desenvolvimento de tecnologias inteligentes para gestão de cidades; (2) porque correm riscos ambientais; (3) para redução de custos da gestão e manutenção das cidades; ou (4) necessidade de melhoria da mobilidade urbana.

Nosso estudo buscou mapear as principais contribuições dos estudos brasileiros sobre o tema cidades inteligentes. Evidenciamos que a maior parte dos estudos são empíricos e buscam, por meio de estudos de caso e estudos experimentais, desenvolver ferramentas que possam contribuir para o 
desenvolvimento de cidades inteligentes e facilitar a vida nos centros urbanos, focando sempre na qualidade de vida da população e sustentabilidade.

Tabela 1 - Algumas das principais contribuições dos estudos brasileiros no avanço das pesquisas sobre cidades inteligentes.

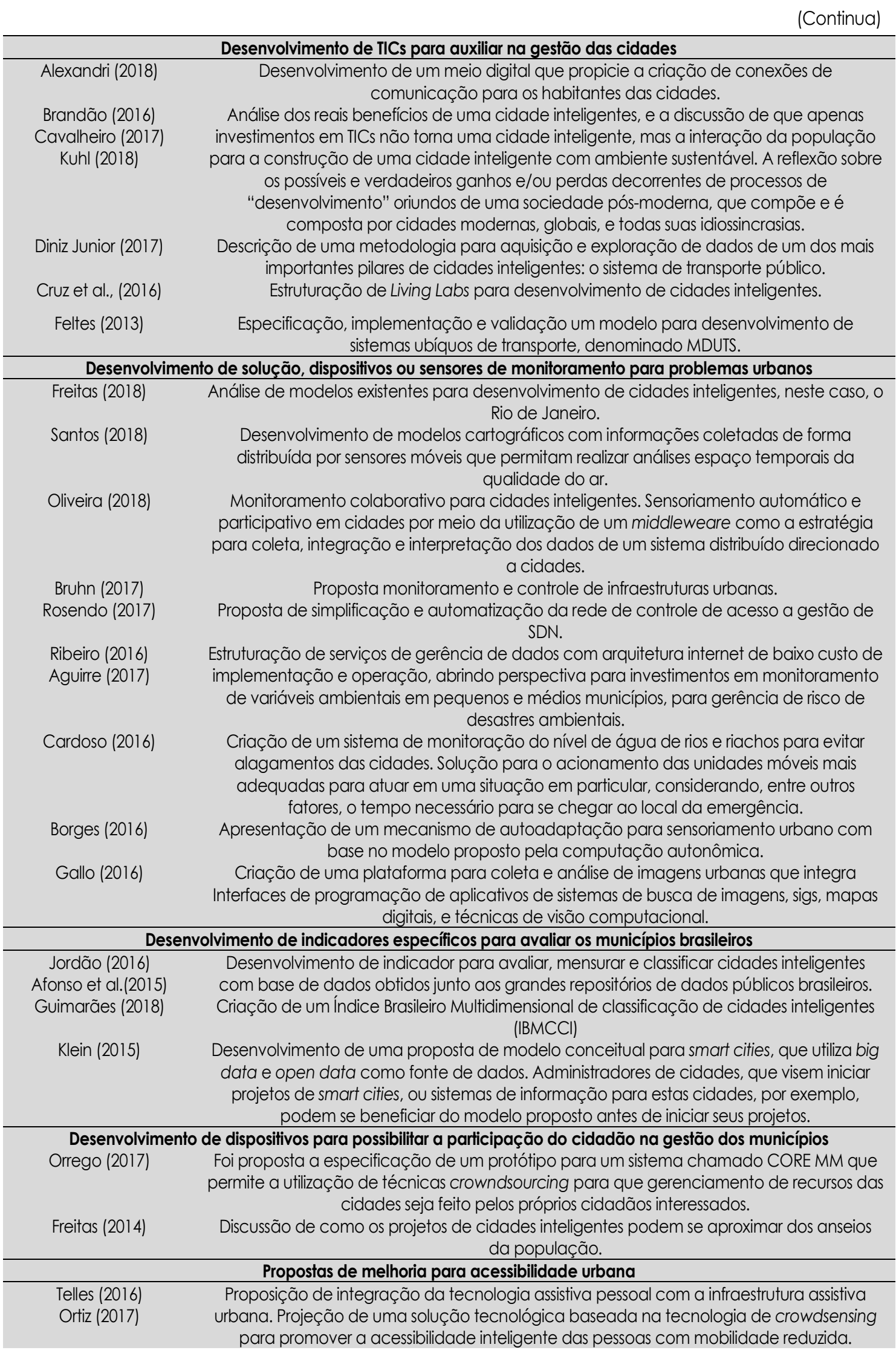


(Conclusão)

\begin{tabular}{|c|c|}
\hline \multicolumn{2}{|r|}{ Utilização de loT para gestão das cidades } \\
\hline Cruz (2017) & $\begin{array}{l}\text { Utilização de loT aplicada em projetos de governança e desenvolvimento de sistemas de } \\
\text { automação autônomos para controle e monitoramento do consumo racional de recursos }\end{array}$ \\
\hline Tomas (2014) & $\begin{array}{c}\text { Especificação, projeção e implementação uma Arquitetura de Software (AS) para Cidade } \\
\text { Inteligente (CI) que permita o desenvolvimento de soluções com base em Internet das } \\
\text { Coisas (loT), independente das especificações de cada tecnologia e características físicas } \\
\text { das cidades. }\end{array}$ \\
\hline \multicolumn{2}{|c|}{ Desenvolvimento de soluções para melhor gestão dos recursos naturais, como a energia, nas smart cities } \\
\hline Rostirolla (2016) & Sugestões para minimizar consumo de energia elétrica no Metro. Proposta de um modelo \\
\hline Moreira Neto (2016) & $\begin{array}{l}\text { Desenvolvimento de um mecanismo preditivo para eleição de coletores semânticos em } \\
\text { grupos de nós sensores sem fio, cujo objetivo central é reduzir o consumo de energia de } \\
\text { uma rede de sensores. Análise da influência da modernização do sistema de controle de } \\
\text { trens no consumo de energia no metrô de São Paulo. Análise técnico-econômica do } \\
\text { impacto da penetração da geração distribuída em redes de distribuição. }\end{array}$ \\
\hline \multicolumn{2}{|c|}{ Desenvolvimento de propostas para melhorar o armazenamento e a privacidade de dados } \\
\hline Silva (2014) & Ao investigar mecanismos de provisão de privacidade propostos em diferentes contextos \\
\hline Ferraz (2016) & $\begin{array}{l}\text { Inteligentes (que essencialmente compõe o que chamamos de Cidades Inteligentes), } \\
\text { constatou-se que não é possível construir uma proposta de implementação de } \\
\text { privacidade que atenda estritamente apenas um paradigma, já que cada um deles tem } \\
\text { a função de cobrir uma parte específica no processo de provisão/consumo de serviços. } \\
\text { Proposta de solução baseada na criação de um ambiente multi-identidade, que permita } \\
\text { manter a privacidade e o anonimato de usuários, ainda que anônimo, dentro de } \\
\text { ambientes interoperáveis e heterogêneos. }\end{array}$ \\
\hline Avelar (2017) & $\begin{array}{l}\text { Apresentação e avaliação propostas para os problemas de armazenamento eficiente de } \\
\text { conteúdo na rede e de suporte à mobilidade do provedor na arquitetura ICN. }\end{array}$ \\
\hline
\end{tabular}
Fonte: Elaborado pelos autores (2018).

Como podemos observar na Tabela 1 os estudos desenvolvidos no Brasil que tratam sobre cidades inteligentes estão mais interessados no desenvolvimento de solução para problemas urbanos, com foco na melhoria da qualidade de vida da população e na sustentabilidade, bem como no desenvolvimento de TICs para auxiliar na gestão das cidades. Podemos destacar como principais contribuições a utilização de IoT para gestão das cidades; o desenvolvimento de dispositivos de monitoramento de problemas urbanos; o desenvolvimento de índices específicos para avaliar os municípios brasileiros; o desenvolvimento de dispositivos para possibilitar a participação do cidadão na gestão dos municípios; o desenvolvimento de propostas para melhorar a privacidade dos dados; sugestões para melhor tomada de decisão referente a gestão das smart cities; o desenvolvimento de soluções para melhor gestão dos recursos naturais, como a energia, nas smart cities, propostas de melhoria para acessibilidade urbana.

\section{Discussões e considerações finais}

O objetivo deste estudo foi identificar a rede de pesquisadores brasileiros que estudam cidades inteligentes, e descrever suas principais contribuições para o debate do tema. Para isso foram coletados dados das universidades, grupos de pesquisa, autores de teses, dissertações e artigo publicados no Brasil que tratam de cidades inteligentes.

Os resultados apontam que existem diversas Universidades interessadas no estudo do tema; 58 universidade públicas e privadas possuem grupos de pesquisa nas mais diversas áreas estudando cidades 
inteligentes. Destacam-se a Universidade de São Paulo (USP), Universidade Federal de Santa Catarina (UFSC), Universidade Tecnológica Federal do Paraná (UTFPR) e Universidade Federal de Sergipe (UFS) com o maior número de grupos de estudo.

Muitos estudos têm sido desenvolvidos nos programas de pós-graduação (PPG) em nível de mestrado e doutorado nas mais diversas áreas, destacam-se, contudo, as áreas das Engenharias (principalmente Engenharia Elétrica), Ciências da Computação e Administração. Há inclusive um PPG em Cidades Inteligentes e Sustentáveis na Universidade Nove de Julho (UNINOVE) no estado de São Paulo. Quatro universidades publicaram o equivalente a 41,33\% das pesquisas brasileiras em cidades inteligentes: Universidade de São Paulo (SP); Universidade Nove de Julho (SP); Pontifícia Universidade Católica de Campinas (SP); e Universidade Federal de Pernambuco (PE), todas as pesquisas são ligadas aos PPGs.

Em nossa análise não foram identificados, no entanto, formação de redes de pesquisa entre os grupos existentes ou os programas de pós-graduação. Considerando que o estudo do tema cidades inteligentes é de interesse público, entende-se que uma maior articulação entre os programas e os grupos de pesquisa poderia beneficiar os estudos e a consolidação das pesquisas brasileiras. Sendo o Brasil um país que possui um território bastante amplo e diversificado geográfica e socialmente torna-se um terreno propício para esses tipos de estudo. Sugere-se inclusive o desenvolvimento de um número maior de smart campus para desenvolvimento destas pesquisas.

Constatamos também que poucos são os estudos que abordam o tema cidades inteligentes disponíveis na base de dados Spell, talvez, por ser uma base mais focada em gestão, enquanto, a maioria das publicações é proveniente de áreas como a Engenharia e Ciências da Computação.

Muitas cidades serviram de objeto desses estudos, a maioria no estado de São Paulo, contudo, os estudos analisados, em sua maioria, não trazem os municípios em que os estudos foram realizados, apenas descrevem com maior profundidade o experimento que realizaram. Foi possível identificar alguns fatores que levam os pesquisadores a escolherem essas cidades para estudos, como: projetos de incentivos ao desenvolvimento de pesquisa em cidades inteligentes, necessidade de combate de algum risco ambiental ou pelo fato da necessidade de redução de custos ou melhoria da mobilidade urbana.

Ao realizarmos a análise sistemática da literatura, uma frase foi recorrente nos estudos analisados: a falta de consenso quanto ao conceito de cidades inteligentes. Freitas (2018), por exemplo, questiona se seria possível haver uma definição derradeira para smart cities, de maneira que contemplasse todos os aspectos abordados nos diversos estudos existentes. Na visão do autor seria extremamente difícil concatenar todos os pontos do debate em apenas uma definição.

Weiss (2019) realizou um estudo em que apresenta e discute a agenda de pesquisas em TIC aplicáveis ao campo da gestão urbana com vistas à materialização das cidades inteligentes, nesse estudo entrevistou 84 pesquisadores, sendo $68 \%$ brasileiros. 0 autor ainda sugeriu três conceitos de cidades inteligentes para que os entrevistados indicassem qual melhor representa a ideia de cidades inteligentes. Esses consideraram o conceito de cidades inteligentes cunhado por Giffinger et al. (2007) como o mais adequado: cidade inteligente tem bom desempenho e atua de forma prospectiva em economia, pessoas, governança, mobilidade, ambiente e condições de vida construídos sobre a combinação inteligente do interesse e atividades de cidadãos conscientes, independentes e aptos a tomar decisões. Adicionalmente, 
busca e identifica soluções que permitam que a cidade moderna melhore a qualidade dos serviços prestados aos cidadãos.

Em nossa análise, enquanto alguns pesquisadores utilizaram como base de seu estudo o conceito de outro autor, alguns arriscaram cunhar seu próprio conceito. Aqueles cujas áreas de estudo envolviam tecnologia utilizaram as TICs como principal argumento que diferencia uma cidade "normal" de uma cidade inteligente. Por exemplo, citamos os conceitos cunhados por Tomas (2014) e Molina (2017). Outros colocam o cidadão como principal agente na transformação de construção de cidades inteligentes, como Guimarães (2018). Outros ainda atentam para a preocupação com a sustentabilidade e os impactos ambientais derivados da vida nas cidades, assim como Diniz Junior (2017).

Os estudos brasileiros trazem contribuições para os estudos no campo, mas em sua maioria a preocupação está em desenvolver estudos para melhoria da qualidade de vida urbana, buscando principalmente minimizar problemas decorrentes de desastres naturais (Cardoso, 2016); privacidade de dados e informações (Silva, 2014; Ferraz, 2016); ferramentas para gestão do grande número de dados de dispositivos, aplicativos (Ribeiro, 2016; Aguirre, 2017); e dados gerados por meio de IoTs (Cruz, 2017). A preocupação com o grande consumo de energia nos centros urbanos trouxe possibilidades para redução do consumo (Moreira Neto, 2016; Gomes, 2017). Além disso, foram realizadas propostas de indicadores específicos para classificação das cidades brasileiras (Jordão, 2016; Afonso et al., 2015; Guimarães, 2018). Alguns estudos preocuparam-se em desenvolver dispositivos com foco na mobilidade, principalmente de pessoas com deficiência (Telles, 2016; Ortiz, 2017; Alperstedt Neto et al., 2018).

Nossas constatações vêm ao encontro do estudo de Weiss (2018), que identificou em entrevista com pesquisadores, brasileiros e estrangeiro, que as áreas de mobilidade urbana e serviços sociais são as dimensões em que esses mais empregam seus esforços e estudos para criar inovações em tecnologias como Big Data e Ciência de Dados, Internet das Coisas e Inteligência Artificial.

Um importante ponto presente nos estudos brasileiros é a discussão se as cidades inteligentes, da forma que se concebem, de fato trarão benefícios para a população ou servirão para segregar ainda mais a população em grupos distintos, os que vivem e os que não vivem em cidades inteligentes (Brandão, 2016; Cavalheiro, 2017; Kuhl, 2018). Diante dessa diversidade de possibilidades de estudo, são sugeridas pesquisas futuras, pois, ainda há longo caminho a ser percorrido no Brasil, tanto na academia quanto em propostas práticas para desenvolvimento de cidades inteligentes. São sugeridos:

- Estudos para desenvolvimento de gerencia de risco integrada com a sociedade (Aguirre, 2017);

- Estudos para comparar projetos de desenvolvimento de cidades inteligentes com outros projetos, sendo implementados em todo o mundo, analisando seus resultados e capacidade de criar valor público (Cavalheiro, 2017);

- Estudos de gestão de projetos no contexto de Living Labs e redes de colaboração e métodos de implementação disponíveis para desenvolvimento dos produtos e serviços nos laboratórios (Cruz et al., 2016);

- Desenvolvimento de pesquisa que envolve Internet das Coisas (IoT) para melhoria da qualidade de vida nas cidades (Cruz, 2017); 
- Estudos que tratem do desenvolvimento de metodologias que valorize a participação da sociedade através de ecossistemas de inovação que incentivem a cocriação de soluções em conjunto com a esfera pública, empresarial e acadêmica para criação das Human Smart Cities (Aune, 2017).

- Estudos que busquem compreender até que ponto os resultados das iniciativas de cidades inteligentes refletem as necessidades e expectativas dos cidadãos e de que maneira os recursos providos pelas iniciativas são convertidos em efetivações pela população (Pereira, 2016).

- Estudos que desenvolvem programas específicos para gestão de cidades inteligentes, que auxiliem a partir de análise de dados a tomada de decisão do gestor público.

Nosso estudo buscou contribuir com a academia propondo um panorama dos estudos desenvolvidos nacionalmente em cidades inteligentes e abrir lacunas para o desenvolvimento de novos estudo, contudo, uma contribuição importante é mostrar por meio das redes onde e quem está escrevendo sobre o tema a fim de incentivar a formação de redes de relacionamento para fortalecimento das pesquisas e desenvolvimento das cidades brasileiras, uma vez que esse é um tema que apresenta um impacto social importante. Algumas limitações foram observadas na condução do estudo, como: dificuldade na análise dos estudos pela diversidade de áreas de conhecimento existentes, o que dificulta a compreensão do autor, também sugerimos que estudos semelhantes sejam realizados com apoio de software para a análise dos dados, uma vez que são diversos e necessitam de análise de conteúdo para tal.

Sugerimos como estudo futuro uma análise com maior profundidade das redes, realização de entrevistas com os líderes de grupos de pesquisa e coordenadores de PPGs buscando, desse modo, alternativas para ampliação das pesquisas em rede em cidades inteligentes. Também sugerimos a elaboração de um estudo de meta-análise sobre cidades inteligentes a fim de identificar o que já foi desenvolvido isoladamente e em que ponto a pesquisa se encontra. Da mesma forma sugerimos que as revistas priorizem de alguma forma as publicações na área, assim como os PPGs incentivem a publicação das teses e dissertações em formato de artigos científicos, pois muitos estudos analisados não foram publicados em formato de artigo.

\section{Referências}

Afonso, R. A., Costa, L. C., Álvaro, A., \& Garcia, V. C. (2015). SCiAl: Usando Dados Públicos para Agrupar Cidades Alagoanas. GESTÃO.Org - Revista Eletrônica de Gestão Organizacional, 13(Especial ed.), 331-339, Recuperado em 12 de novembro de 2019, de http://www.spell.org.br/documentos/ver/41161/scial--usando-dados-publicos-para-agruparcidades-alagoanas/i/pt-br

Aguirre, T. F. A. (2017). Gerência de risco de enchentes em cidades inteligentes (Dissertação de Mestrado). Pontifícia Universidade Católica de Campinas, Campinas.

Alejandro, V.A. \& Norman, A. G. (2005). Manual Introdutório à Análise de Redes Sociais. Mexico: Universidad Autonoma Del Estado de Mexico (UAEM). 2005.

Alexandri, A. (2018). Desafio: transformar Gonçalves (MG) em uma cidade conectada (Dissertação de mestrado). Mestrado Profissional em Produção Jornalística e Mercado, Escola Superior de Propaganda e Marketing, São Paulo.

Alperstedt Neto, C. A., Rolt, C. R. de, \& Alperstedt, G. D. (2018). Acessibilidade e Tecnologia na Construção da Cidade Inteligente. Revista de Administração Contemporânea, 22(2), 291-310. https://dx.doi.org/10.1590/1982-

7849rac2018170295 
Aune, A. (2017). Human Smart Cities - O cenário brasileiro e a importância da abordagem joined-up na definição de Cidade Inteligente (Dissertação de Mestrado). Mestrado em Engenharia Urbana e Ambiental, Pontifícia Universidade Católica do Rio de Janeiro, Rio de Janeiro.

Avelar, E. A. M. (2017). MOBCACHE: mobilidade de provedor e armazenamento eficiente em redes sem fio orientadas a conteúdo (Tese de Doutorado). Pós-Graduação em Ciências da Computação, Universidade Federal de Pernambuco, Recife, Pernambuco.

Batty, M., Axhausen, K.W., Giannotti, F., Pozdnoukhov, A., Bazzani, A., Wachowicz, M., Ouzounis, G., \& Portugali, Y. (2012) Smart cities of the future. The European Physical Journal Special Topics, 214, 481-518. https://doi.org/10.1140/epjst/e2012-01703-3.

Berst, J. (2018). Four Steps to Smart City Success [Viewpoint]. IEEE Electrification Magazine, 6(2), 112-110. https://doi.org/10.1109/MELE.2018.2816849.

Borges, G. A. (2016). Um Mecanismo Abstrato de Autoadaptação para Sistemas de Sensoriamento Urbano (Dissertação de mestrado). Programa de Pós-Graduação em Computação, Universidade Federal do Rio Grande do Sul, Porto Alegre.

Brandão, F. S. N. (2016). Cidades inteligentes e direito à cidade: a atuação das tecnologias da informação e comunicação na produção de duas perspectivas antagônicas de espaço urbano (Dissertação de Mestrado). Faculdade de Comunicação, Universidade de Brasília, Brasília.

Breiger, R. L. (2004). The analysis of social network, In M. Hardy, \& A. Bryman (Eds.), Handbook of Data Analysis (1. Ed., p. 505-526). London: Sage Publications.

Bruhn, J. C. (2017). Proposta de modelo para estruturação de centros de controle integrados em cidades médias (Dissertação de mestrado). Programa de Pós-Gradução em Engenharia de Produção. Universidade Federal do Rio Grande do Sul, Porto Alegre.

Byun, J., Kim, S., As, J., Kim, S., Shin, H-T., \& Kim J-B. (2016). Smart city implementation Models Based on IoT Technology. Advanced Science and Technology Letters, 129, 209-212. http://dx.doi.org/10.14257/astl.2016.129.41

Camboim, G. (2018). The way to make cities smarter: evidences from Europe (Dissertação de Mestrado). Programa de Pós-Graduação em Administração, Universidade Federal do Rio Grande do Sul, Porto Alegre.

Caragliu, A.; Del Bo, C., \& Nijkamp, P. (2011). Smart Cities in Europe. Journal of Urban Technology, 18(2), 65-82. http://dx.doi.org/10.1080/10630732.2011.601117

Cardoso, M. A. R. (2016). Alerta POA: um sistema de comunicação apoiado em redes de sensores sem fio para monitoração do Córrego Itaim (Dissertação de Mestrado). Programa de Pós-Graduação em Engenharia Elétrica, Pontifícia Universidade Católica de Campinas, Campinas.

Cavalheiro, M. B. (2017). Understanding smart tourism destinations: evidence from smart city project implementation in an international tourism destination (Tese de Doutorado). Doutorado em Administração, Fundação Getúlio Vargas, Rio de Janeiro.

Chourabi, H.; Nam, T.; Walker, S.; Gil-Garcia, J. R.; Mellouli, S.; Nahon, K.; Pardo, T. A.; Scholl, H. J. (2012) Understanding Smart Cities: An Integrative Framework. In 45th Hawaii International Conference on System Sciences (p. 2289-2297). Maui. http://dx.doi.org/10.1109/HICSS.2012.615

Cruz, C. E. M., Fonseca, C. B. S., \& Silva, R. B. (2016). Estruturação de Living Labs e sua governança por temas da cidade inteligente: o caso da linha verde em Curitiba (Dissertação de Mestrado). Mestrado Profissional em Gestão e Políticas Públicas, Fundação Getúlio Vargas, São Paulo.

Cruz, M. A. A. (2017). Performance Evaluation of IoT Middleware (Dissertação de Mestrado). Mestrado em Engenharia de Telecomunicações, Instituto Nacional de Telecomunicações, Santa Rita do Sapucaí.

Brasil (2017, 22 de novembro de 2017). Decreto n. 9.203, de 22 de novembro de 2017. Dispõe sobre a política de governança da administração pública federal direta, autárquica e fundacional. Brasília: Presidência da República Secretaria-Geral Subchefia para Assuntos Jurídicos. Recuperado em 16 de julho de 2019, de www.planalto.gov.br/ccivil_03/_Ato2015-2018/2017/Decreto/D9203.htm 
Diniz Junior, P. C. (2017). Serviços telemáticos em uma rede de transporte público baseados em veículos conectados e dados abertos (Dissertação de Mestrado). Programa de Pós-Graduação em Engenharia Elétrica e Informática Industrial Universidade Tecnológica Federal do Paraná, Curitiba.

Feltes, L. H. (2013). MD-UTS: um modelo para desenvolvimento de sistemas ubíquos de transporte (Dissertação de mestrado). Programa Interdisciplinar de Pós-Graduação em Computação Aplicada, Universidade do Vale do Rio dos Sinos, São Leopoldo.

Ferraz, F. S. (2016). IDeM: An Identity-Driven Middleware for interoperable and heterogeneous systems (Tese de Doutorado).Programa de Pós-Graduação em Ciência da Computação, Universidade Federal de Pernambuco, Recife.

Freitas, J. A. (2014). Cidade inteligente búzios: entre paradigmas e percepções (Dissertação de mestrado). Mestrado Acadêmico em História, Política e Bens Culturais, Fundação Getúlio Vargas, Rio de Janeiro.

Freitas, J. A (2018). A invenção da cidade inteligente Rio: uma análise do Centro de Operações Rio pela lente das mobilidades (2010-2016) (Tese de doutorado). Doutorado Acadêmico em História, Política e Bens Culturais, Fundação Getúlio Vargas, Rio de Janeiro.

Gallo, D. S. (2016). Monitoramento colaborativo para cidades inteligentes (Tese de Doutorado). Departamento de Engenharia de Computação e Sistemas Digitais, Escola Politécnica da Universidade de São Paulo, São Paulo.

Giffinger, R. et al. (2007). Smart Cities: Ranking of European Medium-sized Cities. Vienna: Centre of Regional Science, Final report October 2007. Recuperado em 12 de novembro de 2019, de http://www.smart-

cities.eu/download/smart_cities_final_report.pdf

Gomes, F. M. (2017). A influência da modernização do sistema de controle de trens no consumo de energia do metrô de São Paulo (Dissertação de Mestrado). Programa de Mestrado em Cidades Inteligentes e Sustentáveis, Universidade Nove de Julho, São Paulo.

Guimarães, J. G. A. (2018). Cidades Inteligentes: proposta de um modelo brasileiro multi-ranking de classificação (Dissertação de Mestrado). Programa de Pós-Graduação em Administração, Universidade de São Paulo, São Paulo.

Hammi, B., Khatoun, R., Zeadally, S., Fayad, A., \& Khoukhi, L. (2018). IoT technologies for smart cities . IET Networks, 7(1), 1-13. http://dx.doi.org/10.1049/iet-net.2017.0163

Hollands, R. G. (2008). Will the real smart city please stand up? City: analysis of urban trends, culture, theory, policy, action, 12(3), 303-320. https://doi.org/10.1080/13604810802479126

Instituto Brasileiro de Estudos e Pesquisas Sociais (IBEPES) (2015). Institucional. Recuperado em 10 de novembro de 2019, de www.ibepes.org.br/?page_id=11.

Jordão, K. C. P. (2016). Cidades Inteligentes: uma proposta viabilizadora para a transformação das cidades brasileiras (Dissertação de Mestrado). Programa de Pós-Graduação em Sistemas de Infraestrutura Urbana, Pontifícia Universidade Católica de Campinas, Campinas.

Klein, V. B. (2015). Uma proposta de modelo conceitual para uso de big data e open data para smart cities (Dissertação de mestrado). Programa de Pós- Graduação em Engenharia e Gestão do Conhecimento, Universidade Federal de Santa Catarina, Florianópolis.

Kuhl, A. (2018). Developing and applying a smart city for development model: the case of COR in Rio de Janeiro (Dissertação de Mestrado). Mestrado Executivo Em Gestão Empresarial, Fundação Getúlio Vargas, Rio de Janeiro.

Molina, R. (2017). Inovação em empresas de tecnologia de informação para mobilidade urbana sustentável (Dissertação de Mestrado). programa de mestrado profissional em empreendedorismo, Universidade de São Paulo, São Paulo.

Moreira Neto, M. (2016). Flecha: um mecanismo preditivo para eleição de coletores semânticos em grupo de nós sensores sem fio (Dissertação de Mestrado). Programa De Pós-Graduação Em Engenharia De Teleinformática, Universidade Federal do Ceará, Fortaleza.

Nam, T., \& Pardo, T. A. (2011). Conceptualizing Smart City with Dimensions of Technology, People, and Institutions. In Proceedings of the 12th Annual International Conference on Digital Government Research (p. 282-291) Maryland.

Neirotti, P., De Marco, A., Cagliano, A. C., Mangano, G., \& Scorrano, F. (2014). Current trends in Smart City initiatives: Some stylised facts. Cities, 38, 25-36. https://doi.org/10.1016/j.cities.2013.12.010 
Oliveira, A. A. A. M. (2018). Investigate and Analyse a City - INACITY (Dissertação de mestrado). Programa de PósGraduação em Ciências da Computação, Universidade de São Paulo, São Paulo.

Orrego, R. B. S. (2017). CORE-MM: um modelo de crowdsourcing para cidades inteligentes baseada em gamificação (Dissertação de Mestrado). Programa Interdisciplinar de Pós-Graduação em Computação Aplicada, Universidade do Vale do Rio dos Sinos, São Leopoldo.

Ortiz, L. N. (2017). Integração da tecnologia assistiva pessoal com a infraestrutura urbana: uma proposta para cidades inteligentes (Dissertação de Mestrado). Programa de Pós-Graduação em Sistemas de Infraestrutura Urbana, Pontifícia Universidade Católica de Campinas, Campinas.

Pereira, G. V. (2016). Contribuição de Iniciativas de Cidades Inteligentes no Desenvolvimento Humano: Uma Análise da Percepção de Agentes de Centros de Operações Municipais no Brasil (Tese De Doutorado). Programa de Pós-Graduação em Administração, Pontifícia Universidade Católica do Rio Grande do Sul, Porto Alegre.

Pinheiro Junior, L., \& Cavalheiro, L. D. (2017) Smart Cities: A Research Agenda of The Brazilian Smart Cities. Conference Paper. In 14th International Conference on Information Systems \& Technology Management, (p. 1-21). São Paulo: CONTECSI. DOI:10.5748/9788599693131.

Recuero, R. (2017). Introdução à análise de redes sociais online. (1. Ed.). Salvador: EDUFBA.

Ribeiro, A. S. (2016). Cidades digitais e publicação de sistemas em nuvem: uma metodologia para a tomada de decisão (Dissertação de Mestrado). Programa de Pós-Graduação em Engenharia Elétrica, Pontifícia Universidade Católica de Campinas.

Rosendo, D. (2017). A High-Level Authorization Framework For Software-Defined Networks (Dissertação de mestrado). Programa de Pós-Graduação em Ciências da Computação, Universidade Federal de Pernambuco, Recife.

Rostirolla, G. (2016). ElCity: um modelo elástico e multinível de economia de energia para cidades inteligentes (Dissertação de mestrado). Programa Interdisciplinar de Pós-Graduação em Computação Aplicada, Universidade do Vale do Rio dos Sinos, São Leopoldo.

Santos, A. S. (2018). Análise espaço temporal da qualidade do ar em vias urbanas por meio de redes de sensores com nós embarcados em ônibus coletivos (Tese de Doutorado). Departamento de Engenharia de Transportes, Universidade de São Paulo, São Paulo.

Silva, W. M. (2014). GO!SIP: Um framework de privacidade para cidades inteligentes baseado em pessoas como sensores (Dissertação de Mestrado). Pós-graduação em Ciência da Computação, Universidade Federal de Pernambuco, Recife.

Telles, M. J. (2016). MASC: Um modelo computacional para cidades inteligentes assistivas (Dissertação de Mestrado). Programa de Pós-Graduação em Computação Aplicada, Universidade do Vale do Rio dos Sinos, São Leopoldo.

Tomas, G. H. R. P. (2014). Uma arquitetura para cidades inteligentes baseada na internet das coisas (Dissertação de Mestrado). Programa de Pós-graduação em Ciência da Computação, Universidade Federal de Pernambuco, Recife.

Universidade Nove de Julho (2019). Mestrado em Cidades Inteligentes e Sustentáveis: apresentação do programa. Recuperado em 06 de setembro de 2019, de www.uninove.br/mestrado-e-doutorado/programa-de-pos-graduacaoem-cidades-inteligentes-e-sustentaveis-ppg-cis/conheca-o-programa/apresentacao-do-programa/

Weiss, M. C. (2019). Cidades Inteligentes: Uma visão sobre a agenda de pesquisas em tecnologia da informação. Revista Brasileira de Gestão e Inovação - Brazilian Journal of Management \& Innovation, 6(3), 162-187.

https://doi.org/10.18226/23190639.v6n3.08

Zanella, A., Bui, N., Castellani, A., Vangelista, L., \& Zorzi, M. (2014). Internet of Things for Smart Cities. IEEE Internet of Things Journal, 1(1), 22-33. https://doi.org/10.1109/JIOT.2014.2306328

O presente trabalho foi realizado com apoio da Coordenação de Aperfeiçoamento de Pessoal de Nível Superior - Brasil (CAPES) - Código de Financiamento 001.

This study was financed in part by the Coordenação de Aperfeiçoamento de Pessoal de Nível Superior - Brasil (CAPES) - Finance Code 001.

\section{Editor responsável: Fábio Duarte}

Recebido: 14 maio 2018

Aprovado: 19 ago. 2019 